\title{
Use of methylphenidate in the treatment of major depressive disorder in an outpatient mental health center
}

\author{
Carolina Garnier Lacueva*, Juan Castaño, Patricia Alvaro, Rosa Sanchis, David Córcoles, Francisco Portillo, \\ Belen Diaz, Luis Miguel Martin, Antoni Bulbena \\ From $1^{\text {st }}$ International Congress on Neurobiology and Clinical Psychopharmacology and European \\ Psychiatric Association Conference on Treatment Guidance \\ Thessaloniki, Greece. 19-22 November 2009
}

\section{Background}

According to the American Psychiatric Association practice guidelines, if a patient with Major Depressive Disorder (MDD) has not responded or achieved only a partial response after 4-8 weeks of therapy, a dose change, switch to a new drug, or augmentation therapy is recommended [1].

Combined use of standard antidepressants with dopaminergic agents and psychostimulants can lead to accelerate and enhance response if administered early in the course of treatment [2].

\section{Materials and methods}

Using a sample of 100 patients with diagnosis of MDD who have been visited in Barcelona's Sant Martí Sud outpatient mental health center during the year 2008, Sociodemographical (gender, age) and clinical data (present toxic consume, presence of psychiatric background, use of antipsychotics) are analysed with SPSS 15.0 statistical package.

\section{Results}

Methylphenidate is used in 3\% of the sample with an average dose of $20 \mathrm{mg} / \mathrm{d}$. There is a predominancy in the female gender $(66.7 \%)$, a global average age $66.33 \pm$ 7 years. The psychiatric background most frequently found is the presence of previous depressive disorder episodes (66.7\%). None of these patients had toxic abuse nor had been hospitalised.

\section{Conclusions}

The use of metilphedinate is still not frequent in our sample as augmentation strategy of the antidepressive treatment. However initial results show that the combination with metilphenidate can be useful for patients in need of a rapid improvement in depression, particularly in those with chronic treatment-resistant depression but the tolerability of the combination may limit its use [2]. Further investigation using different treatment to achieve remission in patients with major depression is necessary.

Published: 22 April 2010

\section{References}

1. American Psychiatric Association: Practice guideline for the treatment of patients with major depressive disorder (revision). Am J Psychiatry American Psychiatric Association 2000, 157:1-45.

2. Lavretsky Helen, Park Susan, Siddarth Prabha, Kumar Anand Reynolds FCharles III: Methylphenidate-enhanced antidepressant response to citalopram in the elderly: a double-blind, placebo-controlled pilot trial. Am J Geriatr Psychiatry 2006, 14:2.

3. Craig Nelson J, Pikalov A, Berman R: Augmentation treatment in major depressive disorder: focus on aripiprazole. Neuropsychiatri Dis Treat San Francisco 2008, 4(5):937-948.

\section{doi:10.1186/1744-859X-9-S1-S167}

Cite this article as: Lacueva et al:: Use of methylphenidate in the treatment of major depressive disorder in an outpatient mental health center. Annals of General Psychiatry 2010 9(Suppl 1):S167.

Department of Psychiatry, Hospital del Mar, IAPS, Barcelona, Spain 